\title{
Analytical model of bolt shear resistance considering progressive yield of surrounding material
}

\author{
Chen Wen-qiang ${ }^{1} \cdot$ Li Yi-jia $^{1}$
}

Received: 1 August 2021 / Accepted: 13 December 2021

Published online: 04 January 2022

(c) The Author(s) $2021 \quad$ OPEN

\begin{abstract}
Existing analytical models usually fail to match with the actual conditions due to ignoring the nonlinear behavior of the surrounding material reaction force, which changes progressively with the joint shear displacement from elastic stage to yield stage. To tackle this problem, this study proposes a new analytical model to describe the bolt deformation and bolt contribution from elastic stage to plastic stage. The developed model is verified by available experimental direct shear tests of bolted joints and compared with existing models. Then, based on this model, the effects of the joint dilation angle, the bolt installation angle, the friction angle, and the surrounding material strength on bolt contribution are also analyzed and its implication is further discussed. Our results show that the proposed model can precisely describe the evolution of bolt contribution from elastic stage to plastic stage. Compared with surrounding material strength, the augmentation of the joint dilation angle and friction angle is more beneficial to increase the bolt contribution and the optimal installation angle. The work presented is to attempt to provide a reference for the understanding of bolting mechanism of jointed rock mass, the development of bolting theories and the practice of bolting engineering.
\end{abstract}

Keywords Cable bolt $\cdot$ Reaction force $\cdot$ Shear resistance $\cdot$ Axial force $\cdot$ Transverse shear force

\section{Introduction}

Fully grouted bolt is an important technique for fractured rock mass reinforcement, widely used in the slope, tunnel, and dam, and so on. Bolt anchoring is an effective method to improve the mechanical properties of jointed rock mass. When the unstable rock mass slides along the structural plane or has a sliding trend, the rock mass has a strong transverse restraint effect on the bolt body, and the fully grouted bolt has a large shear stiffness, which restricts the sliding deformation or trend of the unstable rock mass along the structural plane. In this process, the compression stress of surrounding grout material, which generates the bolt transverse shear effect (bolt dowel effect), changes gradually and eventually reaches the yield state [1]. Therefore, the transverse shear effect of bolt has a significant contribution to the anchorage strength of jointed rock mass.

Since Bjurstrom conducted experimental studies on the shear resistance of the rock bolting [2], the reinforcement mechanism of bolts has been widely concerned, and many achievements have been achieved, such as the mechanism of the bolt shear resistance [3-5], the interaction mechanism between rock and bolt $[6,7]$, the failure mechanism of bolts $[8,9]$, and the dowel shear effect of bolts $[10,11]$. In addition to the laboratory test, numerical method and theoretical model have also been used to study the mechanical behavior of the bolt in joint. Haile conducted numerical simulations to analyze the effect of the contribution factors (i.e., bolt diameter, bolt inclination angle, and bolt types) on bolt contribution to joint shear strength [12]. Li et al. conducted numerical experiments

Li Yi-jia, liyijia@email.tjut.edu.cn | 'School of Management, Tianjin University of Technology, Tianjin 300384, China. 
on the influence of contribution factors (i.e., concrete strength, bolt installation angle and bolt diameter) on the bolt contribution [13]. Li et al. carried out the numerical experiments of the shear behaviour of cable bolt in FLAC ${ }^{3 D}$ [14]. Nie et al. simulated the shear behaviour of rock bolts based on beam theory and DDA code [15].

Meanwhile, the theory of beams was applied to the mechanical behavior of the bolted joint [16-18]. The interaction between bolt and surrounding material was simulated by a series of Winkler springs. It is supposed that the dowel effect of bolt is activated by a small displacement, which cannot absolutely reflect the large deformation behavior of the bolt and the surrounding material. Moreover, other researchers have proposed the structure mechanical model to simulate the bolt dowel effect and estimate the bolt contribution to the joint strength [14, 19]. The bolt is considered as a statically determinate beam with both ends fixed, and the bolt axial and transverse shear forces are calculated through the structural mechanics theory in this model. However, this model still cannot reflect the progressive deformation mechanism of bolt transverse shear deformation section.

In summary, the previous research has mainly focused on the two aspects: the influence of the factors like the bolt installation angle, the bolt diameter, the bolt pretension, the grout strength, the joint roughness, the joint opening stress etc., on the bolted joint shear resistance behavior; the calculation of joint shear strength contributed by bolt axial force and bolt transverse shear force. Although the existing work is helpful to explore the mechanical action of the bolted joints, the existing research does not provide a wide acceptance constitutive model. Most researchers pay close attention to the calculation of bolt axial and bolt transverse shear forces subjected to joint shear resistance, but they do not pay more attention to the gradual changes of nonlinear interactions between the bolt and the grout material.

As depicted in Fig. 1, a lot of field observations, experimental direct tests and numerical calculations show that the rock bolt will be "s" deformation shape after the bolt reaches its yield strength [20], which is mainly due to the bolt deformation caused by the transverse shear forces and bending moments, causing the reaction of grout material on the compression side of the bolt. However, in the existing analytical models, the reaction force is simply calculated under the condition of the elastic state or yield state of surrounding grout material, while the transition stage from elastic state to yield state is not considered [21]. That is, the reaction force is a sudden change from elastic state to yield state, as shown in Fig. 2. In fact, in the elastic deformation process, the reaction force of grout on bolt progressively augments with the transverse shear displacement, and is non-uniform distribution along

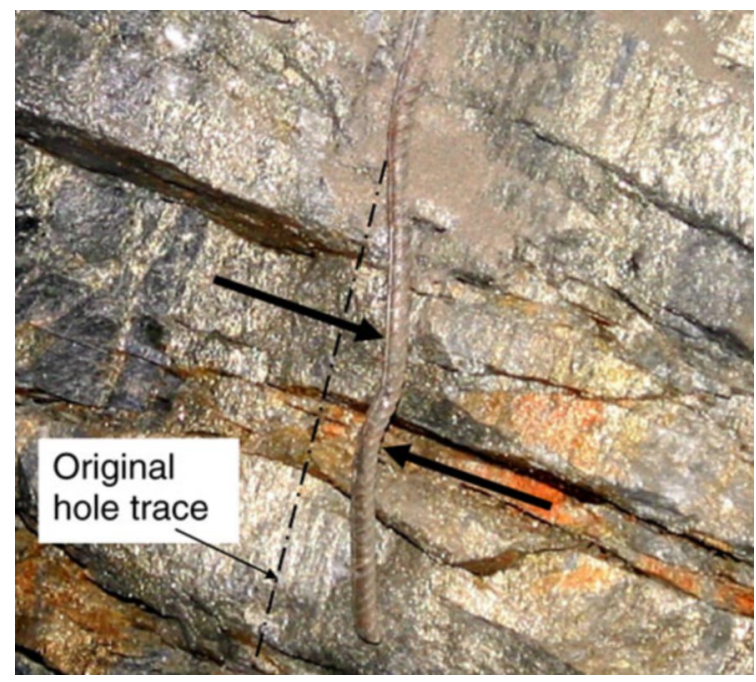

Fig. 1 The shear deformation of bolt in field observation [22]

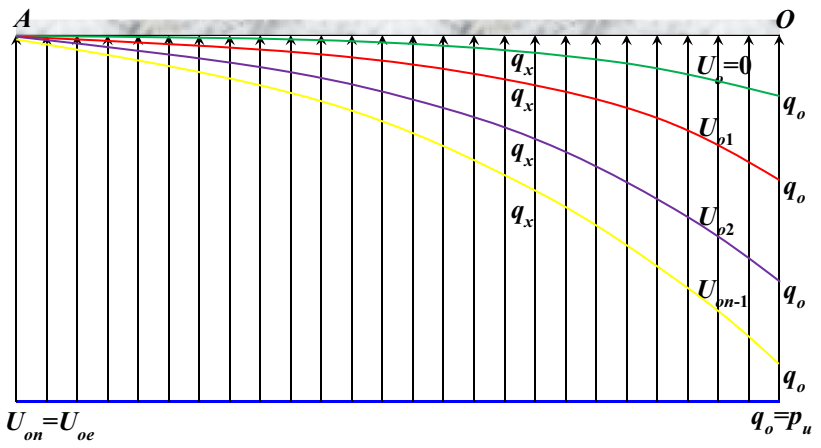

Fig. 2 Deformation mechanism of bolt transverse shear deformation section in previous models

the bolt [1]. As the bolt stress reaches its yield limit value, the reaction force density is evenly distributed along the compression side of the bar. In this process, the length of the surrounding grout reaction force applied to the bolt increases continuously. Therefore, the theoretical model is deduced on the basis of the elastic state or limit state of the reaction force of the surrounding grout on the compression side of the bolt, which will reduce the accuracy of the calculation results.

In addition, during the shearing process, the joint will have the dilatancy effect. Thus, it is must be considered that the perpendicular deformation component to the joint depend on the joint dilation angle. Unfortunately, the influence of this shear dilatancy effect on the axial and transverse deformation of bolt has not been reflected in the existing analytical models.

In this study, we have four main objectives: (1) The theoretical relationships between bolt axial force and bolt axial deformation, and bolt transverse shear force 
and bolt lateral deformation are established from elastic stage to plastic stage considering the progressive yield of surrounding material based on the structure mechanical model; (2) The theoretical model of the bolt contribution to the bolt axial force and the bolt transverse shear force with the shear displacement are proposed in both the elastic and the plastic stages; (3) The developed model is validated by available experimental direct shear tests of bolted joints; (4) According to the proposed model, the effect of the contribution factors (i.e., bolt inclination angle, joint dilation angle, friction angle, and grout strength) on contribution of bolt are systematically analyzed using this proposed analytical model.

Section 2 of this paper introduces the analytical model. Section 3 verifies the proposed analytical model. Section 4 presents the influence analysis of the contribution factors. Section 5 gives the discussion. Section 6 describes the conclusion.

\section{The model}

\subsection{Mechanical behavior of the bolt in elastic domain}

The shear displacement of the joint $U_{o}$ is caused by the shear loads. Meanwhile, the joint dilatancy during shearing will cause the opening displacement of the joint $U_{d}$. Thus, the axial force, $N_{o}$ and transverse shear force, $Q_{o}$ are developed in the bar. At the same time, the axial deformation $u_{0}$ and the transverse deformation $v_{o}$ of the bolt are induced by axial force, $N_{o}$ and transverse shear force, $Q_{0}$ respectively, which makes the bar similar to "s" deformation shape (see Fig. 3). Based on the geometrical relationship between the displacement of the joint and the deformation of the bolt in Fig. 3 , the axial deformation $u_{0}$ and transverse deformation $v_{o}$ on one half of the bolt are deduced.

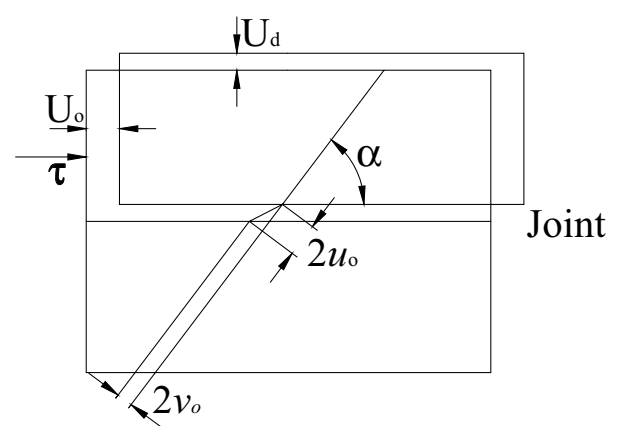

Fig. 3 Sketch of a bolted joint shear deformation
$u_{o}=0.5\left(U_{o} \cos \alpha+U_{d} \sin \alpha\right)$

$v_{o}=0.5\left(U_{o} \sin \alpha-U_{d} \cos \alpha\right)$

The joint dilation angle is denoted as $\beta$, and then the joint opening displacement $U_{d}$ can be expressed as:

$U_{d}=U_{o} \tan \beta$

The reaction force of surrounding grout on the compression side of the bolt is caused by transverse deformation of the bar. It is assumed that the reaction force of grout on the bolt is non-uniform distribution along the direction of the bolt axis, and the reaction force of grout on bolt increases gradually with the transverse deformation, and that the reaction force density is evenly distributed along the axis of the bolt until the rock bolt reaches the yield limit. Point $O$ is the intersection point of the rock bolt and joint. The maximum bending moment of the bolt is at Point $A$, and the rotation and deflection of the bolt are both zero at this location. It also supposed that the length from the joint (point $\mathrm{O}$ ) and the potential plastic hinge of the bolt (point A) progressively increases with the joint displacement. The simplified progressive deformation model of bolt pressure side and the bolt mechanical model are shown in Figs. 4 and 5, respectively. $q_{x}$ is the grout reaction force density on the pressure side of the rock bolt, and $q_{\mathrm{o}}$ is the reaction force density at the point $O$. The reaction force density $q(x)$ can be assumed to a parabolic distribution as follows:

$q(x)=m\left(x^{2}-L^{2}\right)+q_{o}$

where $m$ is a coefficient varying with the joint shear displacement, $m=\left(U_{o e}-U_{o}\right) / U_{o e}$. When $U_{o}=0, m$ is equal to 1 . When the bolt reaches the yield limit $\left(U_{o}=U_{o e}\right), m$ finally approaches zero.

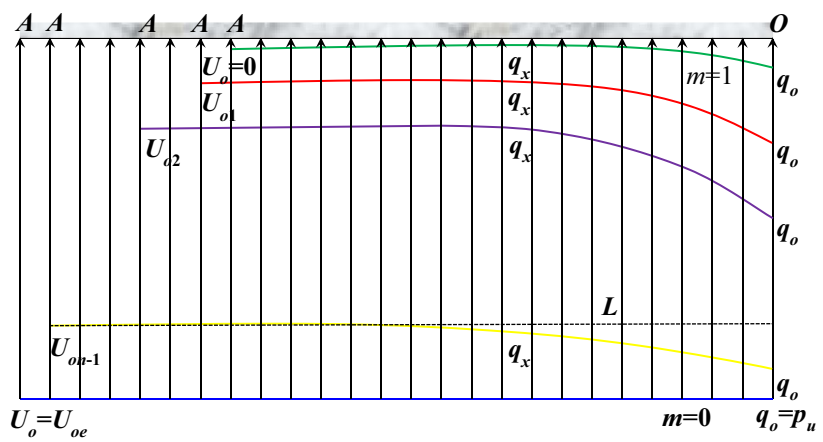

Fig. 4 Reaction force density on the pressure side of bolt before elastic yield limit 


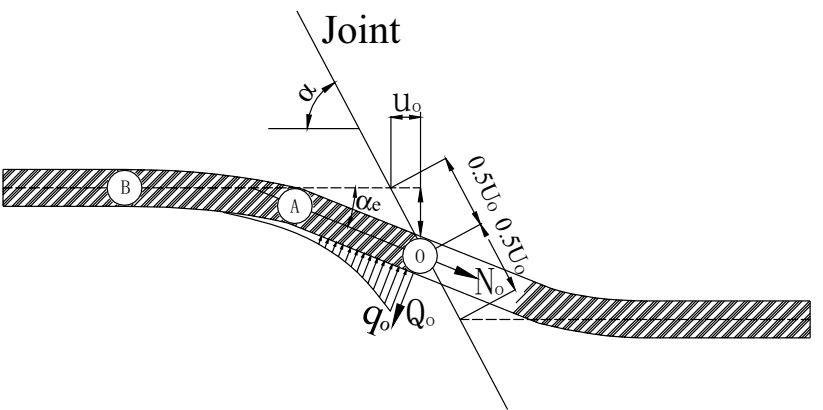

(a)

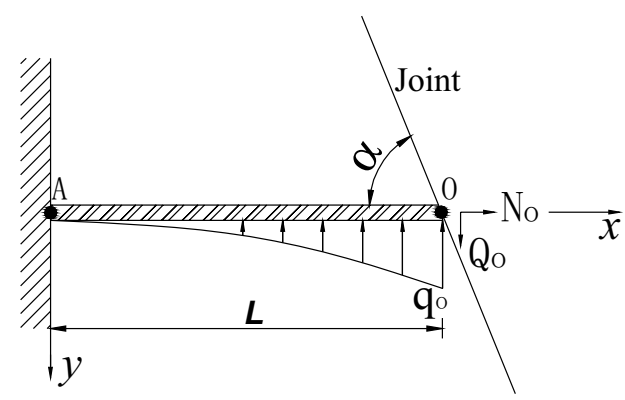

(b)

Fig. 5 Deformation and loading of the bolt for elastic conditions

The reaction force $Q(x)$ and the bending moment $M(x)$ can be obtained by integrating Eq. (4).

$Q(x)=\frac{m}{3} x^{3}-m L^{2} x+q_{o} x$

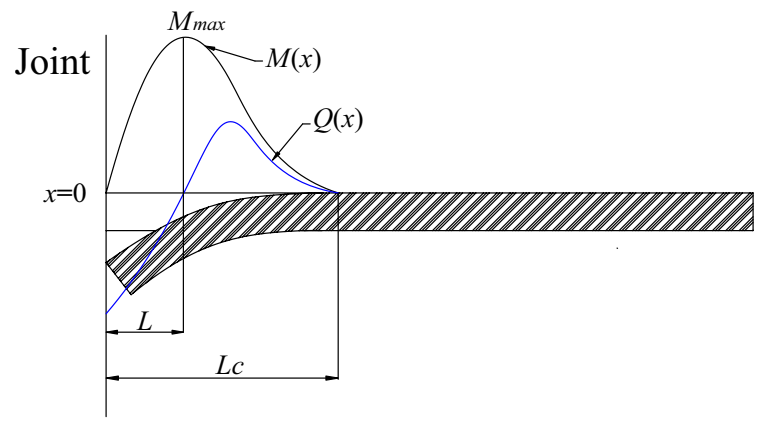

Fig. 6 Location of maximum bending moment and length of curvature zone

To consider the influence of the localized curvature in bolt, the term of the curvature influencing zone, $L_{c^{\prime}}$ is introduced by Maekawa and Qureshi [23], as shown in Fig. 6.

$L_{c}=\frac{3 \pi}{4} \sqrt[4]{\frac{4 E I}{150 \sigma_{c}^{0.85}}}\left[1+3\left(\frac{v_{o}}{D}\right)^{a}\right]$

where $\sigma_{c}$ is the uniaxial compressive strength of grout. $E$ is the bolt elastic modulus. I is the inertia moment of the bolt cross section, equal to $\pi D^{4} / 64$. $D$ is the bolt diameter.

Thus, the location of the maximum bending moment $L$, from the joint, can be calculated by Eq. (8) [23]. The length of $L$ is changed with the transverse deformation

$M(x)=Q_{o}(L-x)-\frac{m x^{4}+6 q_{o} x^{2}-6 m L^{2} x^{2}-12 q_{o} L x+8 m L^{3} x+6 q_{o} L^{2}-3 m L^{4}}{12}$

where $L$ is the length from the joint (point O) (i.e. $x=L$ ) and the potential plastic hinge of the bolt (point A) (i.e. $x=0$ ). Thus, the transverse shear force $Q_{o}=Q(L)=q_{0} L-2 m L^{3} / 3$, the maximum bending moment $M_{A}=M(0)=Q_{0} L-\left(2 q_{0} L\right.$ $\left.{ }^{2}-m L^{4}\right) / 4$ $v_{o}$ of the bolt in elastic conditions.

$L=\frac{L_{c}}{3}$

Based on the approximate differential equation of beam rotation and deflection, the distribution function of rotation and deflection on one side of bolt can be derived from Eq. (6).

$$
\begin{aligned}
\varphi(x)= & -\frac{Q_{o}(L-x)^{2}}{2 E I}-\frac{m x^{5}+10 q_{o} x^{3}-10 m L^{2} x^{3}-30 q_{o} L x^{2}}{60 E I}+20 m L^{3} x^{2}+30 q_{o} L^{2} x-15 m L^{4} x \\
& +\frac{Q_{o} L^{2}}{2 E I}
\end{aligned}
$$




$$
\begin{aligned}
v(x)= & \frac{Q_{o}(L-x)^{3}}{6 E I}-\frac{m x^{6}+15 q_{o} x^{4}-15 m L^{2} x^{4}-60 q_{o} L x^{3}+40 m L^{3} x^{3}++90 q_{o} L^{2} x^{2}-45 m L^{4} x^{2}}{360 E I} \\
& +\frac{Q_{o} L^{2}}{2 E I} x-\frac{Q_{o} L^{3}}{6 E I}
\end{aligned}
$$

where $\varphi(x)$ and $v(x)$ are, respectively, the rotation and deflection equation of the bolt on one side of the joint; when $x=0, \varphi(x)$ and $v(x)$ are both 0 ; when $x=L, \varphi(L)$ and $v(L)$ are, respectively, the bolt rotation angle and the transverse shear displacement at the joint.

The axial displacement of the bolt at the joint $u_{0}$ is caused by the axial force $N_{o}$. Therefore, the axial displacement of the bolt $u_{0}$ can be expressed as:

$u_{o}=\frac{L_{c}}{E A_{b}} N_{o}$

where $A_{b}$ is the area of the bolt cross section, which is equal to $\pi D^{2} / 4$.

Based on Eq. (9), the rotation angle of the bolt $\varphi_{o}$ at the joint can be obtained:

$\varphi_{o}=\frac{L^{2}}{2 E I} Q_{o}-\frac{10 q_{o} L^{3}-4 m L^{5}}{60 E I}$

According to Eq. (10), and considering the influence of shear deformation on $v_{0}$, the transverse displacement $v_{0}$ can be expressed by.

$v_{o}=\left(\frac{L^{3}}{3 E I}+\frac{k L}{G A_{b}}\right) Q_{o}-\left(\frac{L^{4}}{8 E I}+\frac{k L^{2}}{12 G A_{b}}\right) q_{o}+\frac{19 m L^{6}}{360 E I}$

where $k$ is equal to $4 / 3$ [24].

According to Eq. (11) and combining Eq. (12), Eq. (13) and $Q_{o}=Q(L)=q_{0} L-2 m L^{3} / 3$, the axial force, $N_{o}$, transverse shear force, $Q_{o}$ and reaction force density $q_{o}$ at the joint in the elastic deformation stage have the following forms:

$N_{o}=\frac{E A_{b} u_{o}}{L_{c}}$

$Q_{o}=\frac{24 E I G A}{5 G A L^{3}+6 k E I L} v_{o}-\frac{11 m G^{2} A^{2} L^{7}-2 m E I k G A L^{5}-40 m E^{2} I^{2} k^{2} L^{3}}{\left(2 E I k-G A L^{2}\right)\left(75 G A L^{2}+90 k E I\right)}$

$q_{o}=\frac{Q_{O}}{L}+\frac{2 m L^{2}}{3}$

There are two yielding criteria of the bolt [6]: one is the tensile-shear yield of the rock bolt at joint, which is caused by axial force, $N_{o}$ and transverse shear force, $Q_{0}$; the other is the bending yield of the rock bolt at point $A$, which is caused by axial force $N_{o}$ and bending moment $M_{A}$.
$\sigma_{e}=\sqrt{\left(\frac{N_{o e}}{A_{b}}\right)^{2}+3\left(\frac{Q_{o e}}{A_{b}}\right)^{2}}$

$\frac{M_{A}}{1.7 \sigma_{e}\left(\pi D^{3} / 32\right)}+\left(\frac{N_{o}}{\sigma_{e} A_{b}}\right)^{2}=1$

where $N_{o e}$ is the yield axial force at joint, $Q_{o e}$ is the yield transverse shear force at the point $\mathrm{O} ; \sigma_{e}$ is the yield stress of the bar.

\subsection{Mechanical behavior of the bolt after yielding}

After the bolt reaches the yield, the reaction pressure density of the surrounding grout material on the pressure side of the bar quickly reaches the limit value $P_{u}$ (the maximum pressure of the grout reaction per unit length), as shown in Fig. 7. Beyond the yield of the bolt, the section OA of the bolt has the mechanical properties of the truss, so only the bolt axial force, $N_{o}$, increases gradually, and transverse shear force, $Q_{0}$, remains unchanged [25]. When the ultimate strain of the bolt $\varepsilon_{f}$ is reaching, the bolt failure at the joint will be occur. Thus, the bolt failure criteria (point $\mathrm{O}$ ) at the joint caused by the combination of axial force $N_{\text {of }}$ and transverse shear force $Q_{\text {of }}$ can be expressed as:

$\left(\frac{N_{o f}}{N_{p}}\right)^{2}+\left(\frac{Q_{o f}}{Q_{p}}\right)^{2}=1$

where $Q_{o f}$ is equal to $Q_{o e^{\prime}} N_{o p}$ is equal to $A_{b} \sigma_{p^{\prime}} Q_{o p}$ is equal to $A_{b} \sigma_{p} / \sqrt{3}, \sigma_{p}$ is the failure stress of the bolt.

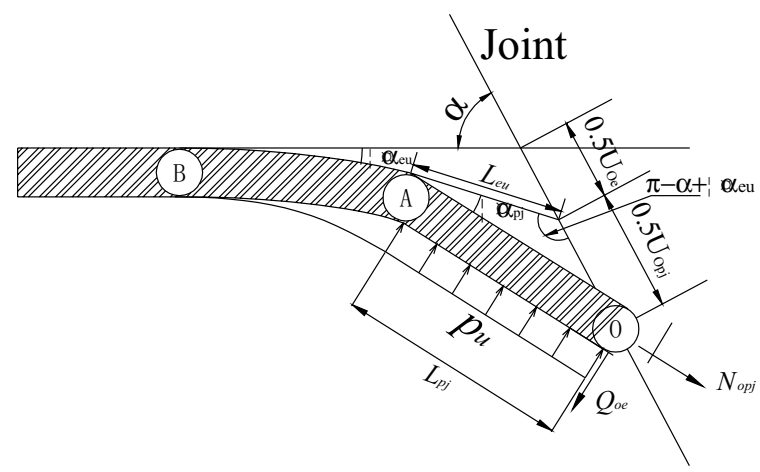

Fig. 7 Deformation and loading of the bolt after yielding 
According to the Eq. (19), $N_{\text {of }}$ is expressed as,

$N_{o f}=N_{p} \sqrt[2]{1-\left(\frac{Q_{o f}}{Q_{p}}\right)^{2}}=N_{p} \sqrt[2]{1-\left(\frac{Q_{o e}}{Q_{p}}\right)^{2}}$

In addition, based on the geometrical consideration in Fig. 7, we can obtain the following equation to correlate the bolt installation angle $a$, the plastic length of the bar $L_{p j}$ and the shear displacement of joint $U_{o p j}$ at step $j$ :

$\frac{0.5 \Delta U_{o p j}}{\sin \Delta \alpha_{p j}}=\frac{L_{e u}}{\sin \left(\alpha-\Delta \alpha_{e u}-\Delta \alpha_{p j}\right)}=\frac{L_{p j}}{\sin \left(\pi-\alpha+\Delta \alpha_{e u}\right)}$

$U_{o p j}=U_{o e}+\Delta U_{o p j}$

$L_{p j}=0.5 \varepsilon_{p j} L_{e u}+L_{e u}$

where $U_{o p j}$ and $\Delta U_{o p j}$ are the globe shear displacement and the shear displacement increment of joint in the plastic stage at step $j . L_{e u}$ and $\Delta a_{e u}$ are the distance between the joint and the plastic hinge (point $A$ ) and the increment rotation of the bolt at the yield, respectively. $L_{p j}$ and $\Delta a_{p j}$ are, respectively, the distance from the joint to the plastic hinge (point $A$ ) and the increment rotation angle of the bolt in plastic stage at step $j . \varepsilon_{p j}$ is the strain of the bolt at step $j$.

From Eq. (21), the increment rotation inclination angle of the bolt in plastic stage $\Delta a_{p j}$ at step $j$ can be expressed as:

$\Delta \alpha_{p j}=\alpha-\Delta \alpha_{e u}-\arcsin \left[\frac{L_{e u} \sin \left(\alpha-\Delta \alpha_{e u}\right)}{L_{p j}}\right]$

Due to the complex of the axial deformation characteristics of the bolt between yield and failure, the description of the development of the bolt axial force is rather difficulty. Based on Neal [26], the diminution of the bolt axial rigidity can be described by a power function. The bolt axial rigidity is related to the plastic strain of the bolt, thus

$K_{j}=A \varepsilon_{p j}^{-B}$

where $K_{j}$ is the axial rigidity of the bolt at step $j, A$ and $B$ are the coefficient, and are discussed in the part 5.

Therefore, the axial force of the bolt after yielding can be expressed as,

$N_{o p j}=N_{o e}+K_{j} \varepsilon_{p j} L_{e u}$

where $N_{o p j}$ is the axial force at step $j$.

According to the Eq. (26), when the strain $\varepsilon_{p j}=\varepsilon_{f}$, the axial force of the bolt $N_{o p j}$ is equal to $N_{o f}$ thus $K_{f}$ can be expressed as:

\section{SN Applied Sciences}

SPRINGER NATURE journal

$$
K_{f}=\frac{N_{o f}-N_{o e}}{\varepsilon_{f} L_{e u}}
$$

\subsection{Bolt contribution to joint shear resistance}

According to the mechanical behavior of the bolted joint, the bolt contribution to the joint shear resistance $T$ should be divided into two components: the bolt contribution to the joint shear resistance provided by the axial force, $T_{N}$ and the bolt contribution to the joint shear resistance provided by the transverse shear force, $T_{Q}$. In this paper, the bolt contribution caused by the transverse shear force is regarded as the dowel effect. The values of $T, T_{N}$ and $T_{Q}$ can be expressed as:

$T_{N}=N_{0}\left\{\sin \alpha \tan \left(\varphi_{b}+\beta\right)+\cos \alpha\right\}$

$T_{Q}=Q_{0}\left\{\sin \alpha-\cos \alpha \tan \left(\varphi_{b}+\beta\right)\right\}$

$T=N_{0}\left\{\sin \alpha \tan \left(\varphi_{b}+\beta\right)+\cos \alpha\right\}+Q_{0}\left\{\sin \alpha-\cos \alpha \tan \left(\varphi_{b}+\beta\right)\right\}$

where $\phi_{b}$ is the joint friction angle.

The superiority degree of the dowel effect, $f$ can be express as the ratio of $T_{Q}$ to $T_{N}$.

$f=\frac{T_{Q}}{T_{N}}$

The $f$ can quantify the advantage of $T_{Q}$ relative to $T_{N}$. $f>1$, the joint shear resistance is almost contributed by the transverse shear force of the bar; $f<1$, the joint shear resistance is almost offered by the axial force of the bar; $f=1$, the shear-resist effect of the two components is the same.

When calculating the shear strength of the bolt in plastic stage, the bolt inclination angle $a$ in Eqs. (28), (29) and (30) should be modified as follow:

$\alpha_{p j}=\alpha-\Delta \alpha_{e u}-\Delta \alpha_{p j}$

Excel Worksheet program is used to calculate the whole process curve of $N_{O^{\prime}} Q_{O}$ and $T$ against the joint shear displacement $U_{o}$ respectively. The specific calculation procedure is shown in Fig. 8.

\section{Model validation}

A series of laboratory direct shear tests of the bolted joints were carried out by Spang $[5,27]$. In order to demonstrate the proposed analytical model, some available direct shear tests of the reinforced joint are used. In his study, 
Fig. 8 The calculation process of the proposed analytical model

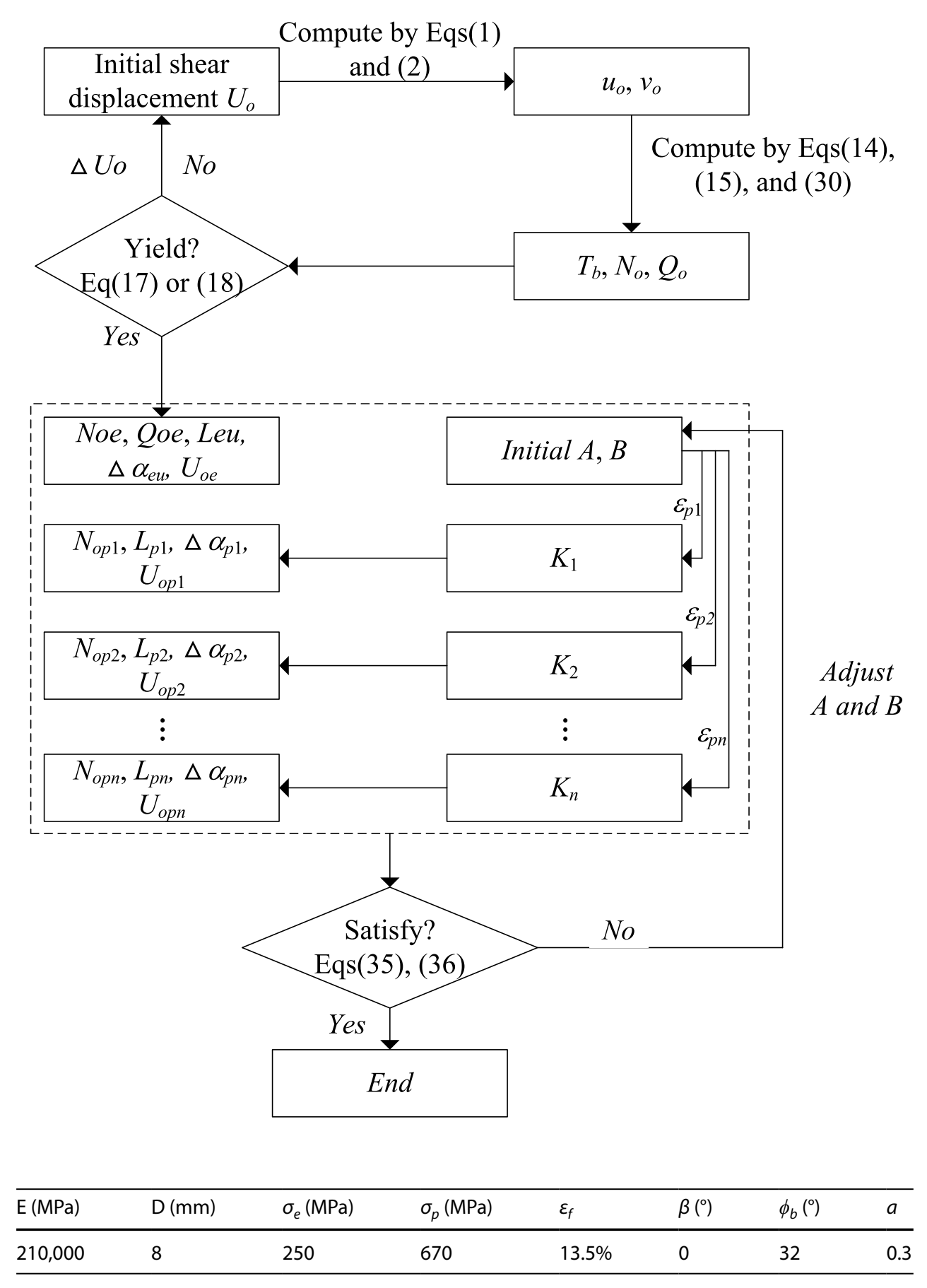

\begin{tabular}{llllllll}
\hline $\mathrm{E}(\mathrm{MPa})$ & $\mathrm{D}(\mathrm{mm})$ & $\sigma_{e}(\mathrm{MPa})$ & $\sigma_{p}(\mathrm{MPa})$ & $\varepsilon_{f}$ & $\beta\left(^{\circ}\right)$ & $\phi_{b}\left({ }^{\circ}\right)$ & $a$ \\
\hline 210,000 & 8 & 250 & 670 & $13.5 \%$ & 0 & 32 & 0.3 \\
\hline
\end{tabular}

Table 1 Mechanical parameters for tests two blocks with the size of $150 \times 150 \times 130 \mathrm{~mm}$ are stacked up to form a specimen with a steel bolt installed across the joint center. The diameter of the bolt is $8 \mathrm{~mm}$. The bolt inclination angles are varied from $60^{\circ}$ to $90^{\circ}$ for shear testing. Granite $\left(\sigma_{c}=105 \mathrm{MPa}\right)$, concrete $\left(\sigma_{c}=40 \mathrm{MPa}\right)$, sandstone $\left(\sigma_{c}=10 \mathrm{MPa}\right)$ are used as block material to simulate the rock. The shear load, the bolt deformation, and the joint shear displacement are recorded during the loading. Based on his experiments, we can obtain the material properties of the bolt and joint directly, as shown in the Table 1. Due to the small thickness of mortar material in the laboratory shear tests, only the rock material is considered as the surrounding material of the bolt in the analytical model.

Figures 9, 10, 11 and 12 show the comparison of the complete curves of shear displacement versus shear load of the bolted joint from the experiments and those from other analytical model without considering progressive yield of surrounding material [27]. The results show that the proposed analytical model is able to precisely predict 


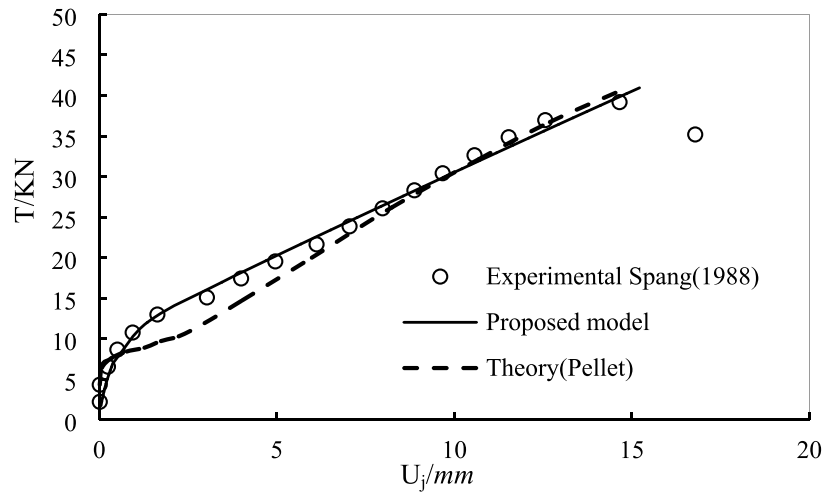

Fig. 9 Joint shear resistance versus joint displacement for a concrete specimen reinforced by a bolt of $8 \mathrm{~mm}$ diameter perpendicular to the joint

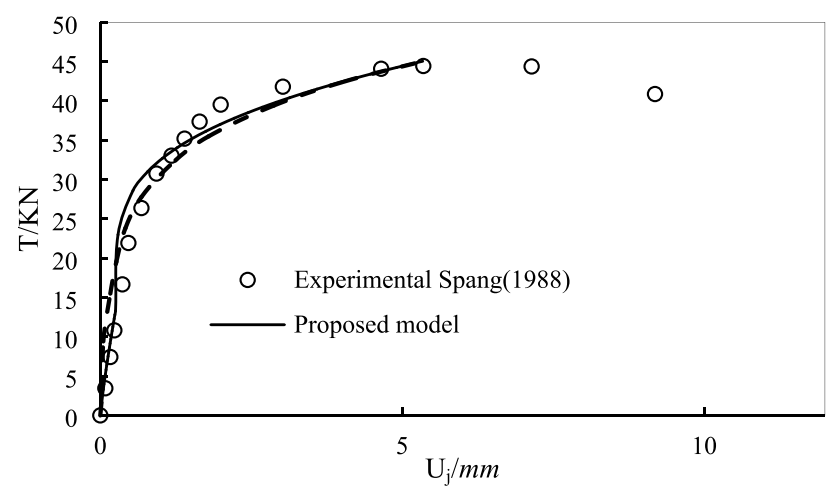

Fig. 10 Joint shear resistance versus joint shear displacement for a concrete specimen reinforced by a bolt of $8 \mathrm{~mm}$ diameter, inclined at 60 degrees

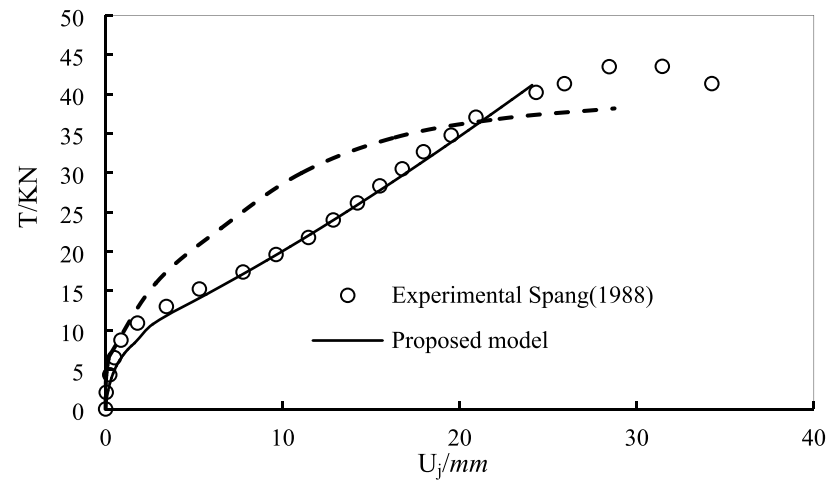

Fig. 11 Bolt contribution versus joint shear displacement for a sandstone specimen reinforced by a bolt of $8 \mathrm{~mm}$ diameter perpendicular to the joint

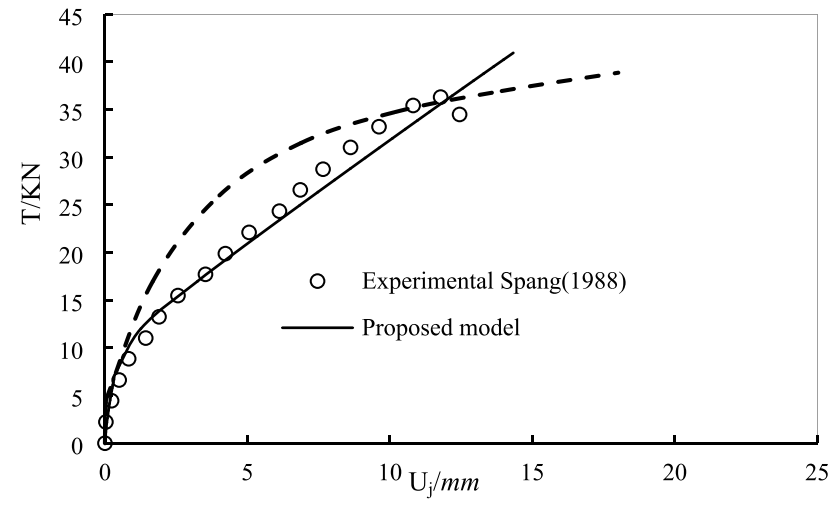

Fig. 12 Joint shear resistance versus joint shear displacement for a granite specimen reinforced by a single bolt of $8 \mathrm{~mm}$ diameter perpendicular to the joint

the bolt contribution evolution for the direct shear tests. Because Pellet did not consider progressive yield of surrounding material and the joint dilatancy effect, when the strength of the surrounding material is large or small, the theoretical calculation by Pellet has a large deviation from the experimental results. Therefore, comparison of the analytical model, the proposed analytical results are more consistent with experimental data. In addition, it can also be concluded in Figs. 9, 10, 11 and 12, that the increasing surrounding material strength could increase the bolt contribution, but reduce the displacement at failure.

The calculation results also show that the bolt yielding meets the tensile-shear yield criteria due to the small diameter of bolt and the hard surrounding material, which is consistent with the conclusion obtained by Ferrero [6]. Ferrero believes that the yielding form of bolt is related to the bolt diameter and the surrounding material strength. The small diameter bolt generally yields by the combination of tensile and shear, while a large diameter bolt may bend yield. The tensile-shear yield occurs in hard material, while the bending yield occurs easily in soft material.

\section{Analysis of the bolt contribution}

In order to analyze the influence of the different factors (i.e., joint dilation angle $\beta$, surrounding material strength $\sigma_{c^{\prime}}$ friction angle $\phi_{b^{\prime}}$, and bolt installation angle $\alpha$ ) affecting the bolt contribution at the yield limit and failure respectively, we carried out a parametric study. The bolt total 
Table 2 Mechanical parameters for calculations

\begin{tabular}{lllllll}
\hline $\mathrm{E}(\mathrm{MPa})$ & $\mathrm{D}(\mathrm{mm})$ & $\sigma_{e}(\mathrm{MPa})$ & $\sigma_{p}(\mathrm{MPa})$ & $\varepsilon_{f}$ & $\phi_{b}\left({ }^{\circ}\right)$ & $a$ \\
\hline 210,000 & 8 & 250 & 670 & $13.5 \%$ & 32 & 0.3 \\
\hline
\end{tabular}

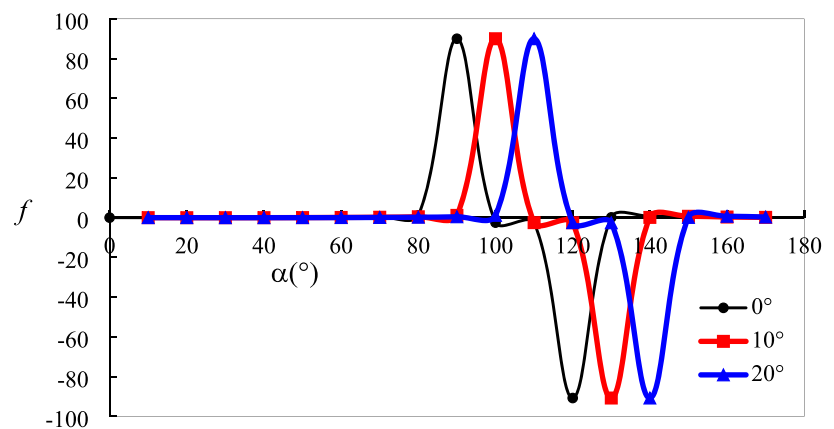

Fig. 13 Influence of $a$ on $f$ for different values of $\beta$

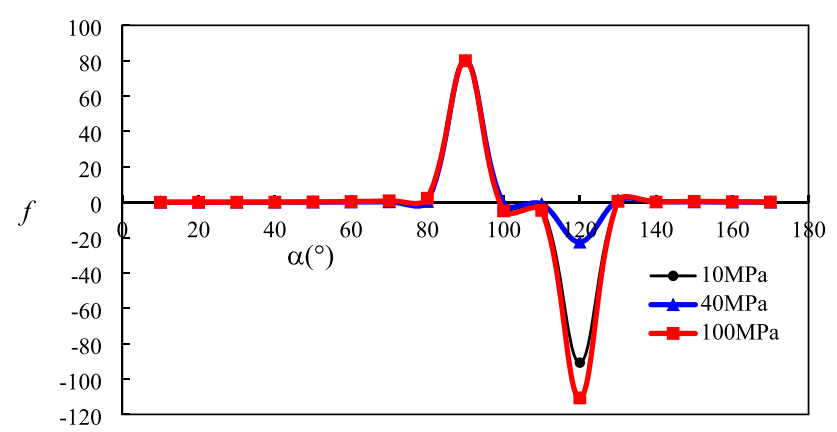

Fig. 14 Influence of $a$ on $f$ for different values of $\sigma_{c}$

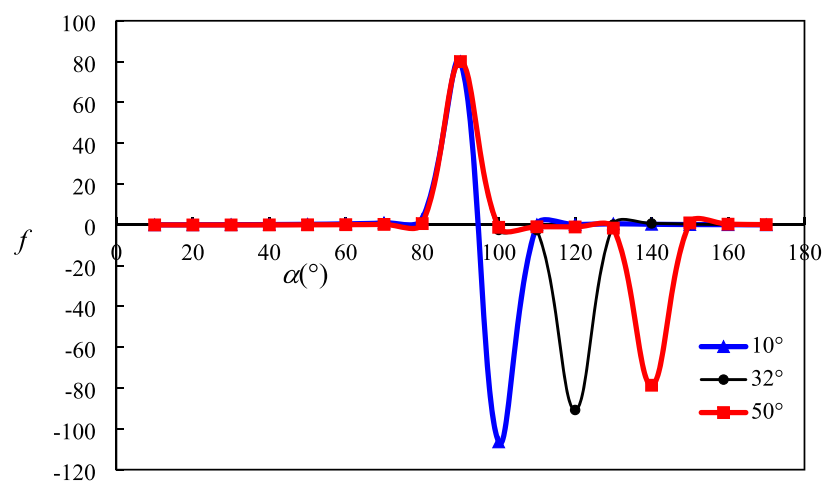

Fig. 15 Influence of $a$ on $f$ for different values of $\phi_{b}$

contribution $(T)$ at the yield limit, $T y$, the bolt total contribution ( $T$ ) at the failure, $T_{f}$, and the ratio of $T_{Q}$ to $T_{N}, f$, at the yield limit were calculated. Thus, we obtained the complete curves of $T y, T_{f}, T_{Q} / T_{N}$ versus bolt installation angle $a$

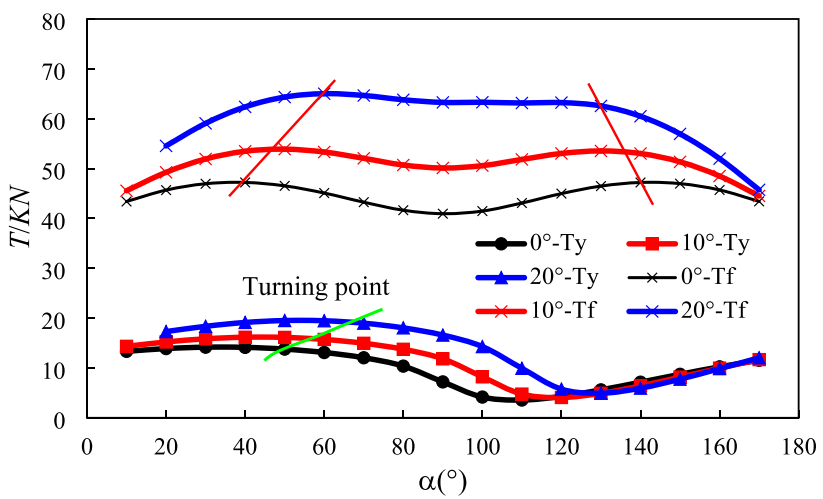

Fig. 16 Influence of $a$ on $T_{y}$ and $T_{f}$ for different values of $\beta$

with different $\beta, \sigma_{c^{\prime}}$ and $\phi_{b}$. The parameters required in the analytical model are shown in Table 2.

\subsection{Influence of bolt installation angle on the ratio $f$}

The effect of the bolt installation angle variation on the ratio $f$ is shown for different values of joint dilation angle in Fig. 13. As can be seen in Fig. 13, for different joint dilation angle, the installation angle has the same width of fluctuation range, in which the dowel effect is more obvious and the dominant of the dowel effect is greatly affected by installation angle, but there is a delay in the position of fluctuation range. For example, when $\beta=0^{\circ}$, the fluctuation range of installation angle is $80^{\circ}<\alpha \leq 130^{\circ}$. When the joint dilation angle increase to $10^{\circ}$, the fluctuation range of installation angle is delayed for $10^{\circ}$, that is, $90^{\circ}<a \leq 140^{\circ}$. The fluctuation range delay value is consistent with the increment of joint dilation angle. When $a \leq 80^{\circ}$, the ratio $f$ is not influenced by the installation angle and joint dilation angle, and $f$ is less than 1 . In other words, the bolt contribution is almost generated by the axial force.

Figure 14 shows the influence of the bolt installation angle $a$ on the ratio $f$ for different values of surrounding material strength. As the surrounding material strength increases, the width and location of fluctuation range remains the same, that is, $80^{\circ}<a \leq 130^{\circ}$. When $110^{\circ}<a \leq 130^{\circ}$, the ratio $f$ increases with the surrounding material strength rising, but under others bolt installation angle, the ratio $f$ is not affected by the surrounding material strength. When $a<80^{\circ}$, and $130^{\circ}<a \leq 180^{\circ}$, the 
ratio $f$ is less than 1 , and not influenced by the installation angle and surrounding material strength.

The influence of the bolt installation angle $a$ on the ratio $f$ for different values of friction angle is shown in Fig. 15. The width of fluctuation range becomes lager when the friction angle increases. For example, when $\phi_{b}=10^{\circ}$, the fluctuation range of installation angle is $80^{\circ}<a \leq 110^{\circ}$. When $\phi_{b}=32^{\circ}$, the fluctuation range of installation angle is $80^{\circ}<a \leq 130^{\circ}$. When $\phi_{b}=50^{\circ}$, the fluctuation range of installation angle is increased to $80^{\circ}<a \leq 150^{\circ}$. When $a<80^{\circ}$, and $150^{\circ}<a \leq 180^{\circ}$, the ratio $f$ is less than 1 , and not influenced by the installation angle and friction angle. In fluctuation range of installation angle, the dowel effect can be improved by increasing the friction angle.

From Figs. 13, 14 and 15, the evolution curve has two regions: weak impact range and fluctuation range. In the weak impact range, the axial force mobilized in the bolt is more obvious, and the ratio is almost not influenced by the joint dilation angle, friction angle, and surrounding material strength. However, in the fluctuation range, the dowel effect becomes obvious, but the width, location, and peak value of the fluctuation range influenced by the joint dilation angle, friction angle, and surrounding material strength. The joint dilation angle will affect the position of the fluctuation range, which leads to the delay of the fluctuation range. The surrounding material strength will affect the peak value of the fluctuation range. The friction angle will affect the peak value and width of the fluctuation range.

\subsection{Influence of bolt installation angle on the bolt contribution}

The influence of the bolt installation angle $a$ on the bolt contribution at yield limit $T_{y}$ and failure $T_{f}$ for different values of joint dilation angle is shown in Fig. 16. At the yield limit, the bolt contribution evolution with the

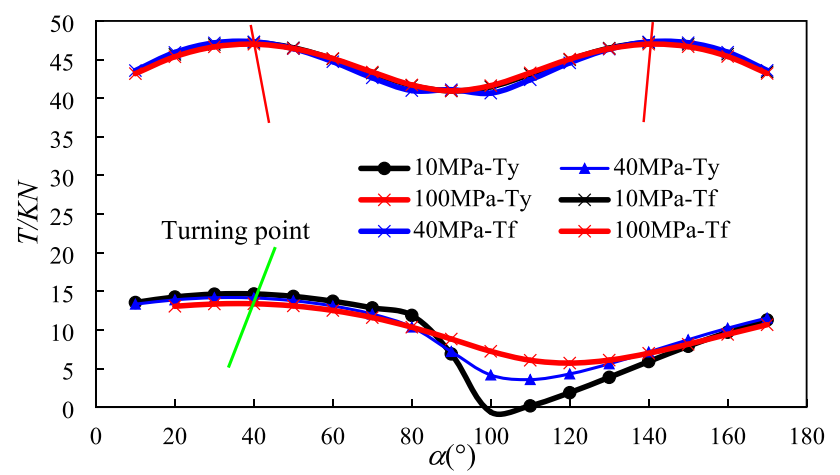

Fig. 17 Influence of $a$ on $T_{y}$ and $T_{f}$ for different values of $\sigma_{c}$ installation angle can be divided into two regions: peak region $\left(0^{\circ}<a \leq 120^{\circ}\right)$ and rise region $\left(120^{\circ}<a \leq 180^{\circ}\right)$. In peak region, as the joint dilation angle increases, the bolt contribution gets lager. However, in rise region, the joint dilation angle bolt does not influence the bolt contribution. In peak region, the turning point (a maximum bolt contribution point) is not a fixed value but increases with the increase of joint dilation angle. The increment of the optimal angle is almost equal to that of the dilation angle. In rise region, the bolt contribution increases linearly with the augmentation of the bolt installation angle.

At failure, the bolt contribution evolution with the installation angle can be divided into three regions: rise region, parabolic region and descending region. At the same installation angle, with the augmentation of the joint dilation angle, the bolt contribution is larger. In the rise region, the bolt contribution increases with the increase of the installation angle. In the parabolic region, when $a=90^{\circ}$, the bolt contribution reaches a minimum value, and the installation angle corresponding to the lowest point does not change with the joint dilation angle. In the descending region, the bolt contribution decreases with the augmentation of the installation angle. With the increase of joint dilation angle, the slope of the curve has a large change rate in the rise and descending regions, while the curve becomes smoother in the parabolic region.

The influence of the bolt installation angle on the bolt contribution at the yield limit $T_{y}$ and the failure $T_{f}$ for different values of surrounding material strength is shown in Fig. 17. At the yield limit, the bolt contribution evolution with the installation angle can also be divided into peak region and rise region. When $90^{\circ}<a \leq 140^{\circ}$, the bolt contribution is greatly influenced by the surrounding material strength, and increases with the increase of surrounding material strength. On the other hand, the bolt contribution is not obviously affected by surrounding material strength for other installation angles. The turning point (a maximum bolt contribution point) is a

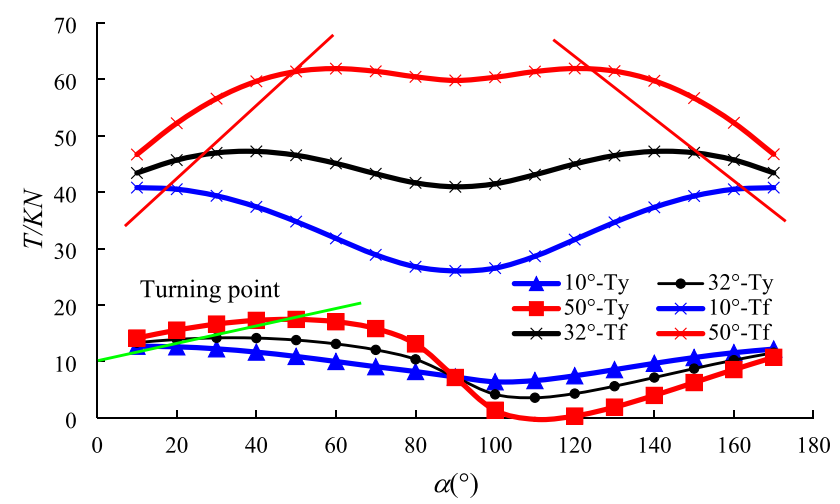

Fig. 18 Influence of $a$ on $T_{y}$ and $T_{f}$ for different values of $\phi_{b}$ 
fixed value, not influenced by the surrounding material strength.

At the failure, the surrounding material strength $\sigma_{c}$ does not affect the finial contribution of the bolt. It can be found from Fig. 17 that with the decrease of the surrounding material strength, the ultimate shear resistances of the bolt have the same value. The reason for this is that the compressive strength of surrounding material will affect the reaction force on the bolt per unit length $P_{u}$ (Eq. 33). $P_{u}$ will improve the bolt contribution provided by the transverse shear force. Based on Eq. 33, we can conclude that the greater the compressive strength of material is, the greater the reaction force on the bolt per unit length $P_{u}$ is, that is, the greater the bolt contribution at the yield limit. However, in the plastic stage, when the reaction force reaches the maximum value, the transverse shear force in bolt will not be improved, so the surrounding material strength will not affect the finial bolt contribution.

$P_{u}=n \sigma_{c} D$

The influence of the bolt installation angle $a$ on the bolt contribution at the yield limit $T_{y}$ and the failure $T_{f}$ for different values of friction angle $\phi_{b}$ is shown in Fig. 18. At the yield limit, the curve of bolt contribution to the installation angle can also be divided into two regions. In the peak region, when $a=90^{\circ}$, the friction angle $\phi_{b}$ has little influence on the bolt contribution. When $0^{\circ}<a \leq 90^{\circ}$, the bolt contribution increases with the augmentation of the friction angle $\phi_{b}$, but decreases with increasing the friction angle $\phi_{b}$ when the installation angle ranges from $90^{\circ}$ to $180^{\circ}$. The turning point is also not a fixed value but increases with the augmentation of friction angle $\phi_{b}$.

At failure, the bolt contribution evolution with the installation angle can also be divided into three regions: rise region, parabolic region and descending region. With the augmentation of friction angle $\phi_{b}$, the installation angle range of rise region and descending region increases, while the installation angle range of region parabola region decreases. And the bolt contribution increases with the augmentation of the friction angle, whatever of the bolt orientation is.

According to Figs. 16, 17 and 18, the curves of the resistant contribution of the bolt over the change of joint dilation angle, surrounding material strength and friction angle have nearly the same shape characteristics in the cases of yield and failure limit of bar. Compared with the joint dilation angle and friction angle, the surrounding material strength has little influence on the bolt contribution at yield limit $T_{y}$ and the failure $T_{f}$. But with the augmentation of joint dilation angle and friction angle, the contribution of bolt can be easily mobilized.

The change of surrounding material strength does not affect the optimal installation angle, but with the augmentation of friction angle and joint dilation angle, the optimal installation angle increases. The optimum installation angle between the joint and the bolt is approximately equal to the joint friction angle. The curves of failure shear resistance of the bolted joint over the change of joint dilation angle, surrounding material strength and friction angle have an almost symmetric distribution. The symmetry point is located near $90^{\circ}$.

\section{Discussion}

The proposed analytical model offers an effective methodology for predicting the bolt contribution to joint strength-shear displacement curves. The limitation of the model is that the joint shear strength is not considered. Due to the interaction between bolt and joint in the process of shear loading, the influence of joint shear strength on axial force and transverse shear force should be considered in the model.

Although there are many researchers have analyzed the hinge point location $L$, but they judge the value $L$ (i.e., $2-3$ times bolt diameter) based on experiments. Jalalifar and Aziz derived $L$ according to the bolt yield criterion (Eq. 34) [20]. However, the value of $L$ gradually decreases with the shear displacement, which is inconsistent with the actual situation. In this study, we introduce and modify the term of the curvature influencing zone, $L_{c}$ and the hinge point location, $L$. Before yielding, the hinge point location, $L$, gradually increases with the shear displacement (Eqs. 7 and 8). However, there is an uncertain parameter $a$ in the formula. According to the existing literature, $a$ is taken as 0.8 [23]. After calculation, it is found that the value of $a$ is related to the bolt diameter, installation angle, surrounding material strength and other factors, so it is necessary to further investigate.

$L=0.025 D^{2}\left(\frac{\sigma_{e}}{\sigma_{c} v_{o}}\right)^{0.5}$

In the previous models, the bolt axial deformation has not been considered properly. For instance, the influence of the joint dilation angle and the increment rotation on the bolt axial deformation is not properly considered. In addition, the bolt axial deformation is not considered after yielding in most analytical models. Therefore, we calculate the bolt axial deformation by taking into consideration the joint dilation angle and the increment rotation from elastic 
stage to plastic stage. However, the bolt-grout interface bond stress is ignored in our model, thus the bolt axial force in the bolt axis does not change.

In the plastic hardening stage, the diminution of the bolt axial rigidity is described by a power function, which is verified by the experimental tests results. However, the bolt axial rigidity (Eq. 25) is related to two uncertain parameter $A$ and $B$. Since we can calculate $K_{f}$ if we know $K_{1}$ (the axial rigidity of the bolt at step 1 ), it is easy to obtain $A$ and $B$. However, there is very little discussion about $K_{1}$. In our study, we use the programming solver to calculate $A$ and $B$ by setting constraint and objective function:

$$
\text { Subject to: } K_{n}=K_{f}
$$

The following constraints should be satisfied:

$$
\left\{\begin{array}{l}
\frac{\left(T_{b p n-1}-T_{b p n-2}\right) /\left(U_{o p n-1}-U_{o p n-2}\right)-\left(T_{b p n}-T_{b p n-1}\right) /\left(U_{o p n}-U_{o p n-1}\right)}{\left(T_{b p n}-T_{b p n-1}\right) /\left(U_{o p n}-U_{o p n-1}\right)} \leq 0.03 \\
\frac{\left(T_{b p n-2}-T_{b p n-3}\right) /\left(U_{o p n-2}-U_{o p n-3}\right)-\left(T_{b p n-1}-T_{b p n-2}\right) /\left(U_{o p n-1}-U_{o p n-2}\right)}{\left(T_{b p n-1}-T_{b p n-2}\right) /\left(U_{o p n-1}-U_{o p n-2}\right)} \leq 0.03 \\
B \geq 0.5
\end{array}\right.
$$

where $n$ is the number of intervals between elastic limit and failure, $T_{b e u}$ and $T_{b p 1}$ are, respectively, the bolt contribution at elastic limit and the bolt contribution in plastic stage at step 1.

\section{Conclusion}

In this paper, an analytical model of bolted rock joint is proposed to predict the bolt contribution evolution, the bolt axial force evolution and the bolt transverse shear force evolution. In our model, we take into account the progressive yield characteristic of surrounding material. In addition, the joint dilation angle and the increment rotation are considered from the elastic stage to the plastic stage. The proposed model agrees well with the available experimental results. The results show that the proposed model can predict accurately the evolution of the bolt contribution, the bolt axial force, and the transverse shear force from elastic stage to plastic stage. Moreover, parametric studies were conducted about four main contribution factors including joint dilation angle, bolt installation angle, friction angle and surrounding material strength, based on the proposed analytical model. Our results show that the above improvements of the model are more consistent with the actual conditions of the bolt deformation.

Acknowledgements This study was financially supported by the National Natural Science Foundation of China through Grants 51809194 and Open Research Fund of State Key Laboratory of Simulation and Regulation of Water Cycle in River Basin (China
Institute of Water Resources and Hydropower Research), Grant No: IWHR-SKL-201817.

Author contributions W.C. designed this study, proposed the method, analyzed the results, and wrote this manuscript; Y.L. revised this manuscript. All authors have read and approved the final manuscript.

\section{Declarations}

Conflict of interest The authors declare no competing interests.

Open Access This article is licensed under a Creative Commons Attribution 4.0 International License, which permits use, sharing, adaptation, distribution and reproduction in any medium or format, as long as you give appropriate credit to the original author(s) and the source, provide a link to the Creative Commons licence, and indicate if changes were made. The images or other third party material in this article are included in the article's Creative Commons licence, unless indicated otherwise in a credit line to the material. If material is not included in the article's Creative Commons licence and your intended use is not permitted by statutory regulation or exceeds the permitted use, you will need to obtain permission directly from the copyright holder. To view a copy of this licence, visit http://creativecommons. org/licenses/by/4.0/.

\section{References}

1. Holmberg $M$ (1991) The mechanical behaviour of untensioned grouted rock bolts. Ph.D. thesis, Royal Institute of Technology, Stockholm

2. Bjurstrom S (1974) Shear strength of hard rock joints reinforced by grouted untensioned bolts. In: Proceedings of the 3rd international conference ISRM congress, Denver, USA

3. Haas CJ (1981) Analysis of rock bolting to prevent shear movement in fractured ground. Min Eng 33(6):698-704

4. Ludvig B (1983) Shear tests on rock bolts. In: rock bolting: theory and application in mining and underground construction, proceedings of the international symposium on rock bolting. Abisko, pp 193-203

5. Spang K, Egger P (1990) Action of fully-grouted bolts in jointed rock and factors of influence. Rock Mech Rock Eng 23(3):201229. https://doi.org/10.1007/BF01022954

6. Jalalifar H, Aziz N (2010) Analytical behaviour of bolt-joint intersection under lateral loading conditions. Rock Mech Rock Eng 43:89-94. https://doi.org/10.1007/s00603-009-0032-6

7. Grasselli G (2005) 3D behaviour of bolted rock joints: experimental and numerical study. Int J Rock Mech Min Sci 42(1):13-24. https://doi.org/10.1016/j.ijrmms.2004.06.003

8. LiYZ, Liu CH (2019) Experimental study on the shear behavior of fully grouted bolts. Constr Build Mater 223:1123-1134. https:// doi.org/10.1016/j.conbuildmat.2019.06.207

9. Jalalifar H, Aziz N, Hadi M (2006) The effect of surface profile, rock strength and pretension load on bending behaviour of fully grouted bolts. Geotech Geol Eng 24:1203-1227. https://doi.org/ 10.1007/s10706-005-1340-6

10. Ferrero AM (1995) The shear strength of reinforced rock joints. Int J Rock Mech Min Sci Geomech Abstr 32(6):595-605. https:// doi.org/10.1016/0148-9062(95)00002-X

11. Ferrero AM, Giani GP, Migliazza M, Egger P (1997) Physical and numerical analysis of reinforced rock joint behaviour. Rivista Italiana di Geotecnica 3:63-89 
12. Haile A (1999) A mechanistic evaluation and design of tunnel support systems for deep level South African mines. PhD thesis, University of Natal, Durban, South Africa

13. Li L, Hagan PC, Saydam S, Hebblewhite B (2016) Li Y (2016) Parametric study of rockbolt shear behaviour by double shear test rock. Rock Mech Rock Eng 49(12):4787-4797. https://doi.org/ 10.1007/s00603-016-1063-4

14. Li XW, Nemcik J, Mirzaghorbanali A, Aziz N, Rasekh H (2015) Analytical model of shear behaviour of a fully grouted cable bolt subjected to shearing. Int J Rock Mech Min Sci 80:31-39. https:// doi.org/10.1016/j.ijrmms.2015.09.005

15. Nie W, Guo W, Ma SQ, Zhao ZY (2020) Numerical modelling of fully grouted rockbolts subjected to shear load. Rock Mech Rock Eng 53(5):2493-2503. https://doi.org/10.1007/ s00603-020-02049-8

16. Stimpson B (1987) An analytical method for determining shear stiffness of an inclined grouted bolt installed across on open discontinuity. Int J Min Geo-Eng 5(3):299-305

17. Sq MA, Zhao ZY, Peng J, Gui Y (2018) Analyticalmodeling of shear behaviors of rockbolts perpendicular to joints. Constr Build Mater 175:286-295

18. Ma SQ, Zhao ZY, Shang JL (2019) An analytical model for shear behaviour of bolted rock joints. Int J Rock Mech Min Sci 121:104019. https://doi.org/10.1016/j.ijrmms.2019.04.005

19. Liu CH, Li YZ (2017) Analytical study of the mechanical behavior of fully grouted bolts in bedding rock slopes. Rock Mech Rock Eng 50(2):413-2423. https://doi.org/10.1007/ s00603-017-1244-9
20. Jalalifar $\mathrm{H}, \mathrm{Aziz} \mathrm{N}$ (2010) Experimental and 3D numerical simulation of reinforced shear joints. Rock Mech Rock Eng 43:95-103. https://doi.org/10.1007/s00603-009-0031-7

21. Zhao ZH, Sun W, Chen SJ, Wang WM, Wang QB (2020) Coupling model of jointed rock mass and rock bolt in offshore LPG underground storage. Energy Sci Eng 8:1468-1483

22. Li CC (2010) Field observations of rock bolts in high stress rock masses. Rock Mech Rock Eng 43(4):491-496

23. Maekawa K, Qureshi J (1996) Computational model for reinforcing bar embedded in concrete under combined axial pullout and transverse displacement. Proc JSCE 31(538):227-239. https://doi.org/10.2208/jscej.1996.538_227

24. Gere JM, Timoshenko SP (1990) Mechanics of materials. Kent Publishing Company, Amsterdam

25. Pellet F, Egger P (1996) Analytical model for the mechanical behaviour of bolted rock joints subjected to shearing. Rock Mech Rock Eng 29:73-97. https://doi.org/10.1007/BF01079755

26. Neal BG (1977) The plastic methods of structural analysis. Wiley, New York

27. Pellet $F$ (1994) Strength and deformability of jointed rock masses reinforced by rock bolts. Ph.D. thesis, Swiss Federal Institute of Technology, Lausanne, Switzerland

Publisher's Note Springer Nature remains neutral with regard to jurisdictional claims in published maps and institutional affiliations. 